\title{
The Clausius-Mossotti Formula and Its Nonlocal Generalization for a Dielectric Suspension of Spherical Inclusions
}

\author{
B. U. Felderhof, ${ }^{1}$ G. W. Ford, ${ }^{2}$ and E. G. D. Cohen ${ }^{3}$
}

Received May 17, 1983

\begin{abstract}
Employing a recently developed cluster expansion for the effective diclectric constant of a suspension of spherical inclusions, we show which parts of the cluster integrals give rise to the Clausius-Mossotti formula. The same selection of terms is then used to obtain an approximate expression for the wave-vectordependent effective dielectric tensor. For a system of hard spheres with only dipole polarizability this expression is evaluated in closed form. This last result is then used to derive the form of the electrostatic potential due to a point charge in the effective medium. For physically reasonable values of the polarizability, the potential has asymptotically the form corresponding to a medium with the Clausius-Mossotti dielectric constant, while at short range it oscillates about this form. For values of the polarizability beyond the physical range critical points are found at which the oscillations become long range.
\end{abstract}

KEY WORDS: Clausius-Mossotti formula; cluster expansion; random media; dielectric; suspectibility; nonlocal.

\section{INTRODUCTION}

As is well known, the concept of an effective dielectric constant, though extremely useful for the description of the macroscopic electrical behavior of a polarizable medium, nonetheless has only limited validity. When the average Maxwell electric field varies rapidly in space it becomes necessary to take the nonlocality of the dielectric response into account. Two circumstances in which this effect of spatial dispersion is important have been

\footnotetext{
Institut für Theoretische Physik A, RWTH Aachen, 5100 Aachen, West Germany.

${ }^{2}$ The University of Michigan, Ann Arbor, Michigan.

${ }^{3}$ The Rockefeller University, New York, New York.
} 
studied extensively. ${ }^{(1)}$ Both types of situation occur in solid state systems, where either the discreteness of the crystal lattice ${ }^{(2-4)}$ or the motion of the excitons $^{(5)}$ may cause the response to be nonlocal. In this paper we study a third cause of spatial dispersion, namely, the finite size of the polarizable particles. We consider in particular a dielectric suspension of spherical inclusions in a uniform medium. Analogous conclusions hold on a more microscopic scale when the particles are considered to be polarizable molecules.

In a recent paper, ${ }^{(6)}$ referred to here as I, we derived a new cluster expansion for the effective dielectric constant and for the effective wavevector-independent susceptibility of a suspension of nonoverlapping spherical inclusions. Our first aim in this present paper is to identify those contributions to the cluster integrals which give rise to the ClausiusMossotti formula for the effective dielectric constant. We do this in Section 3 where we show that a selection of terms corresponding to overlapping configurations of the inclusions leads to the Clausius-Mossotti formula. Our motivation for this selection comes first from the results we obtained in a second recent paper, ${ }^{(7)}$ referred to here as II, in which we studied in detail the two-inclusion cluster integrals, which correspond to the secondorder terms in the density expansion of the effective dielectric constant. There we saw that the overlap contribution was exactly the corresponding term in the density expansion of the Clausius-Mossotti formula and that in general the remaining contributions are relatively small. We are therefore led to suppose that the overlap contribution will also be the largest in the terms of higher order in the density.

In Section 3 we also remark upon the analogy between our selection of terms and that made in the dynamical derivation of the Enskog correction to the Boltzmann equation. There a similar selection gives the largest contribution. As is well known, the Clausius-Mossotti formula gives a remarkably good approximation to the effective dielectric constant of a polarizable medium of nonoverlapping spherical inclusions. ${ }^{(8,9)}$ We feel our results shed light on the nature of these approximations (ClausiusMossotti, Enskog, and others) in which a selection of terms gives the largest contribution to the exact result.

The arguments we give in Section 3 which lead one to expect that the terms neglected in the Clausius-Mossotti formula should be small apply as well to the case of finite wave vector. We are led therefore to make the same selection of terms in the cluster expansion for the wave-vectordependent effective susceptibility. This approximation yields a plausible extension of the Clausius-Mossotti formula to nonzero wave vector. In Section 4 we calculate the susceptibility in this approximation for a model of finite spheres with pointlike polarizability at their center. For this model, 
which describes exactly what are called the dipole-dipole interactions of the inclusions, the results can be expressed in closed form. As an application of these results, in Section 5 we evaluate the electrostatic potential due to a point charge in the medium with inclusions. The form we find for the potential, which at short distance deviates from the Coulomb potential corresponding to a point charge in a uniform medium, illustrates the effects of nonlocal response described by the wave-vector-dependent susceptibility.

As a preliminary, in order to make this paper self-contained, and also because due to the requirements of proof the description in I was rather long and involved, we recapitulate in the following section the prescription for forming the cluster expansion of the susceptibility tensor.

\section{THE CLUSTER EXPANSION OF THE SUSCEPTIBILITY TENSOR}

We consider a system of identical spherical inclusions embedded in a uniform background with dielectric constant $\epsilon_{1}$. The inclusions are assumed to be statistically distributed with a probability distribution which is spatially homogeneous, isotropic, and independent of the applied field. In addition, the inclusions are not allowed to overlap, i.e., the probability distribution vanishes if the separation of any pair of inclusions is less than their diameter. The average (over the distribution of inclusions) polarization $\langle\mathbf{P}\rangle$ and the average electric field $\langle\mathbf{E}\rangle$ are in general linearly related:

$$
\langle\mathbf{P}\rangle=\mathrm{X} \cdot\langle\mathbf{E}\rangle
$$

where $X$ is the electric susceptibility operator. This operator is in general nonlocal so that, exhibiting explicitly the dependence upon vector indices and field points, the above relation can be written

$$
\left\langle P_{j}(\mathbf{r})\right\rangle=\sum_{\mathbf{k}=1}^{3} \int d \mathbf{r}^{\prime} X_{j k}\left(\mathbf{r}-\mathbf{r}^{\prime}\right)\left\langle E_{k}\left(\mathbf{r}^{\prime}\right)\right\rangle
$$

That the susceptibility kernel $X_{j k}$ is a function only of the difference $\mathbf{r}-\mathbf{r}^{\prime}$ follows from our assumption of spatial homogeneity in the distribution of scatterers. In I we obtained an irreducible cluster expansion for the susceptibility operator:

$$
\mathrm{X}=\sum_{s=1}^{\infty} \frac{1}{(s-1) !} \mathbf{X}_{s}
$$

where the sum is over the number of inclusions in the cluster, and $X_{s}$ can be expressed in the form

$$
\mathbf{X}_{s}=\int \cdots \int d \mathbf{R}_{1} \cdots d \mathbf{R}_{s} \sum_{(B)} b(B) \mathrm{C}(B)
$$


Here the integration is over the positions of the centers of the $s$ inclusions and the sum is over all ordered partitions of the position labels $1, \ldots, s$ into disjoint subsets with the condition that the label 1 be in the first subset. We represent a partition of the labels into $k$ disjoint subsets by $(B)$ $\equiv\left(B_{1}\left|B_{2}\right| \ldots \mid B_{k}\right)$ in which the slashes indicate the partitioning, with $B_{1}$ the first subset, $B_{2}$ the second, $\ldots$, and $B_{k}$ the $k$ th. Within the summation $b(B)$ is the block distribution function and $C(B)$ is the chain operator. We describe the formation of these quantities below.

Our scheme for forming the terms within the summation in (2.4) is based on the lattice of ordered partitions of the set of labels $1,2, \ldots, s$. This is an arrangement of the partitions in rows according to the number of disjoint subsets. Thus for $s=2$, the lattice consists of two rows,

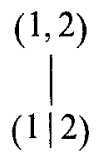

and for $s=3$,

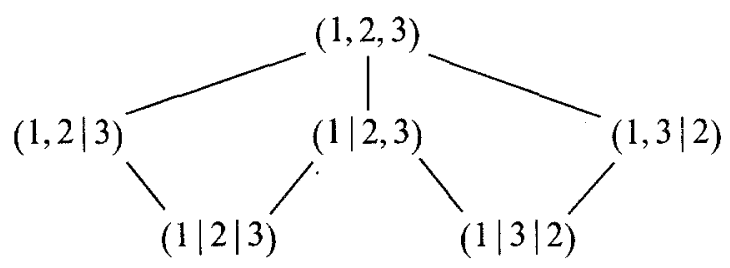

As indicated in these examples, each partition in the lattice is associated with those partitions in the row above which are obtained by merging adjoining subsets, i.e., by removing a slash. This results in a partial ordering of the partitions (denoted by $\leqslant$ ) with $(B) \leqslant\left(B^{\prime}\right)$ if the partition $\left(B^{\prime}\right)$ is either the same as $(B)$ or can be obtained from $(B)$ by removing one or more slashes. For example, in the lattice for $s=3,(1|2| 3) \leqslant(1 \mid 2,3)$ and $(1|2| 3) \leqslant(1,2,3)$ but there is no ordering between $(1|2| 3)$ and $(1,3 \mid 2)$.

The block distribution functions are expressed in terms of the familiar partial distribution functions which characterize the distribution of the inclusions. The latter are defined such that

$$
n(1,2, \ldots, s) d \mathbf{R}_{1} d \mathbf{R}_{2} \ldots d \mathbf{R}_{s} \equiv n\left(\mathbf{R}_{1}, \mathbf{R}_{2}, \ldots, \mathbf{R}_{2}\right) d \mathbf{R}_{1} d \mathbf{R}_{2} \ldots d \mathbf{R}_{s}
$$

is the probability of finding a configuration of $s$ inclusions, the center of one in volume element $d \mathbf{R}_{1}$ about the position $\mathbf{R}_{1}$, that of another in $d \mathbf{R}_{2}$ about $\mathbf{R}_{2}, \ldots$, and that of another in $d \mathbf{R}_{s}$ about $\mathbf{R}_{s}$. Here we have indicated our shorthand notation in which we show only the labels of the coordinates specifying a configuration. The rule for formation of the block 
distribution functions is now simply

$$
b(B)=\sum_{\left(B^{\prime}\right) \geqslant(B)}(-)^{k^{\prime}-1} n\left(B_{1}^{\prime}\right) n\left(B_{2}^{\prime}\right) \ldots n\left(B_{k^{\prime}}^{\prime}\right)
$$

where the sum is over the given partition $(B)$ together with those partitions in the rows above which can be obtained from it by removing slashes and $k^{\prime}=k\left(B^{\prime}\right)$ is the row number of $\left(B^{\prime}\right) \equiv\left(B_{1}^{\prime}\left|B_{2}^{\prime}\right| \ldots \mid B_{k^{\prime}}^{\prime}\right)$. For example,

$$
b(1|2| 3)=n(1,2,3)-n(1,2) n(3)-n(1) n(2,3)+n(1) n(2) n(3)
$$

The key property of the block distribution functions is that they vanish for any configuration in which the coordinates whose labels are to the right of a slash are far from those whose labels are to the left. As shown in I, and as can be seen in the illustration above, this follows from the product property of the partial distribution functions: for a configuration in which the coordinates are in widely separated groups the distribution function is equal to the product of the distribution functions for each group.

The chain operators are expressed ultimately in terms of the operators that give the electric field resulting when a fixed configuration of inclusions is placed in an applied field. Using our shorthand notation, these latter operators are defined by

$$
\mathbf{E}(1,2, \ldots, s)=\mathrm{K}(1,2, \ldots, s) \cdot \mathbf{E}_{0}
$$

Here $\mathbf{E}_{0}$, which is not necessarily uniform, is the applied field, due to a given source distribution of charge in the uniform medium in the absence of inclusions, while $\mathbf{E}(1,2, \ldots, s)$ in the field due to the same source but in the presence of exactly $s$ inclusions (one at $\mathbf{R}_{1}$, another at $\mathbf{R}_{2}, \ldots$, and another at $\mathbf{R}_{s}$ ). The chain operators are most simply expressed in terms of cluster operators based on the operators K. These are of two kinds, the first of which is defined successively as follows:

$$
\begin{aligned}
K(\varnothing) & =L(\varnothing) \equiv 1 \\
K(1) & =L(1)+L(\varnothing) \\
K(1,2) & =L(1,2)+L(1)+L(2)+L(\varnothing)
\end{aligned}
$$

and so on. Here $\varnothing$ denotes the empty set, so $K(\varnothing)$ is the identity dyadic 1 . The general rule is that the operator $K$ corresponding to a given set of labels is written as the sum of operators $L$ corresponding to all subsets.

The second kind of cluster operator involves the susceptibility $\chi(1)$ of an inclusion centered at $\mathbf{R}_{1}$, defined such that

$$
\mathbf{P}(1 ; 2, \ldots, s)=\chi(1) \mathbf{E}(1,2, \ldots, s)=\chi(1) \mathrm{K}(1,2, \ldots, s) \cdot \mathbf{E}_{0}
$$

is the polarization (dipole moment per unit volume) within the inclusion centered at $\mathbf{R}_{1}$ when a configuration of $s$ inclusions (centered at $\mathbf{R}_{1}$, 
$\mathbf{R}_{2}, \ldots, \mathbf{R}_{s}$ ) is placed in the applied field $\mathbf{E}_{0}$. For convenience we take $\mathbf{P}(1 ; 2, \ldots, s)=0$ outside the inclusion centered at $\mathbf{R}_{1}$. We then define successively

$$
\begin{aligned}
\chi(1) \mathrm{K}(1) & =\mathrm{M}(1) \\
\chi(1) \mathrm{K}(1,2) & =\mathrm{M}(1 ; 2)+\mathrm{M}(1) \\
\chi(1) \mathrm{K}(1,2,3) & =\mathrm{M}(1 ; 2,3)+\mathrm{M}(1 ; 2)+\mathrm{M}(1 ; 3)+\mathrm{M}(1)
\end{aligned}
$$

and so on. The general rule is that the operator $\chi(1) \mathrm{K}(1, \ldots, s)$ is written as the sum of operators $M$ corresponding to those subsets of the labels $1, \ldots, s$ which contain the label 1 . The rule for formation of the chain operator is

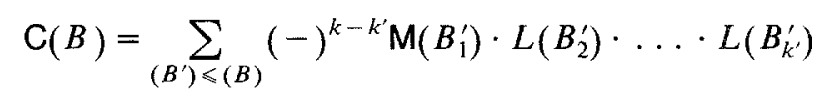

where the sum is over the given partition $(B)$ together with those partitions in the rows below from which it can be obtained by removing slashes. Within the sum, $k$ is the row number of $(B)=\left(B_{1}\left|B_{2}\right| \ldots \mid B_{k}\right)$ and $k^{\prime}$ is the row number of $\left(B^{\prime}\right)=\left(B_{1}^{\prime}\left|B_{2}^{\prime}\right| \ldots \mid B_{k^{\prime}}^{\prime}\right)$. For example,

$$
\mathrm{C}(1 \mid 2,3)=\mathrm{M}(1) \cdot \mathrm{L}(2,3)-\mathrm{M}(1) \cdot \mathrm{L}(2) \cdot \mathrm{L}(3)-\mathrm{M}(1) \cdot \mathrm{L}(3) \cdot \mathrm{L}(2)
$$

The key property of the chain operators, demonstrated in $I$, is that they vanish whenever the coordinates corresponding to labels within one of the subsets are widely separated.

We now turn to the wave-vector-dependent susceptibility, which is introduced as follows. Consider average fields of plane wave form,

$$
\langle\mathbf{E}(\mathbf{r})\rangle=\left\langle\mathbf{E}_{\mathbf{q}}\right\rangle e^{i \mathbf{q} \cdot \mathbf{r}}, \quad\langle\mathbf{P}(\mathbf{r})\rangle=\left\langle\mathbf{P}_{\mathbf{q}}\right\rangle e^{i \mathbf{q} \cdot \mathbf{r}}
$$

Then on account of the assumption of homogeneity of the distribution of inclusions the relation (2.1) can be written

$$
\left\langle\mathbf{P}_{\mathbf{q}}\right\rangle=x^{*}(\mathbf{q})\left\langle\mathbf{E}_{\mathbf{q}}\right\rangle
$$

where $\chi^{*}(\mathbf{q})$ is the wave-vector-dependent electric susceptibility tensor. In I we showed, using standard methods, the relationship of $\chi^{*}$ to the susceptibility operator $X$, defined in (2.1), to get the cluster expansion:

$$
\chi^{*}(q)=\sum_{s=1}^{\infty} \frac{1}{(s-1) !} \sum_{(B)} \int \cdots \int d \mathbf{R}_{2} \ldots d \mathbf{R}_{s} b(B)(\mathbf{q}|\mathbf{C}(\mathbf{B})| \mathbf{q})
$$

in which we have used the notation

$$
\left(\mathbf{q}|O| \mathbf{q}^{\prime}\right) \equiv \int d \mathbf{r} \int d \mathbf{r}^{\prime} e^{-i \mathbf{q} \cdot \mathbf{r}} O\left(\mathbf{r}, \mathbf{r}^{\prime}\right) e^{i \mathbf{q}^{\prime} \cdot \mathbf{r}^{\prime}}
$$

for the plane-wave matrix elements of an operator $O$ with kernel $O\left(\mathbf{r}, \mathbf{r}^{\prime}\right)$. Note that only the diagonal matrix elements of the chain operators occur in 
(2.16). Then, on account of the homogeneity assumption, the integrals are independent of $\mathbf{R}_{1}$ which is held fixed during the integration. The expansion (2.16) will be the basis for our subsequent discussion.

\section{THE CLAUSIUS-MOSSOTTI FORMULA}

We consider now the case where the electric field in the system is uniform. The effective dielectric constant $\epsilon^{*}$ is then given by

$$
\epsilon^{*} 1=\epsilon_{1} 1+4 \pi \chi^{*}(0)
$$

where $\chi^{*}(0)$ is the susceptibility tensor at zero wave vector, and is therefore a multiple of the identity dyadic. In this case it is known from experiment ${ }^{(8)}$ and from work on exact upper and lower bounds ${ }^{(10,11)}$ that a very good approximation to $\epsilon^{*}$ is $\epsilon_{\mathrm{CM}}$ given by the Clausius-Mossotti formula:

$$
\frac{\epsilon_{\mathrm{CM}}-\epsilon_{1}}{\epsilon_{\mathrm{CM}}+2 \epsilon_{1}}=\frac{4 \pi n \alpha}{3 \epsilon_{1}}
$$

where $n$ is the number density of inclusions and $\alpha$ is the dipole polarizability of a single inclusion. Our aim in this section will be to show how the Clausius--Mossotti formula can be obtained by a selection of terms in the expansion (2.16) for $\chi^{*}$.

Putting the cluster expansion (2.16) in (3.1) we can write

$$
\begin{aligned}
\epsilon^{*} & =\epsilon_{1}+\sum_{s=1}^{\infty} \frac{1}{(s-1) !} \sum_{(B)} \epsilon^{*}(B) \\
& =\epsilon_{1}+\epsilon^{*}(1)+\epsilon^{*}(1,2)+\epsilon^{*}(1 \mid 2)+\cdots
\end{aligned}
$$

where

$$
\epsilon^{*}(B) \mathbf{1}=4 \pi \int \cdots \int d \mathbf{R}_{2} \ldots d \mathbf{R}_{s} b(B)(0|\mathrm{C}(B)| 0)
$$

is the contribution corresponding to the partition $(B)$ in the lattice of ordered partitions of $s$ labels. In II (Ref. 7) we considered in detail the two-inclusion contributions $\epsilon^{*}(1,2)$ and $\epsilon^{*}(1 \mid 2)$. There we found that in general $\epsilon^{*}(1 \mid 2)$ is much greater than $\epsilon^{*}(1,2)$ (see Table I of Ref. 7) and, moreover, the entire contribution of $\epsilon^{*}(1 \mid 2)$ comes from the region of integration in which the inclusion at $\mathbf{R}_{2}$ overlaps the inclusion centered at $\mathbf{R}_{1}$, i.e., $\left|\mathbf{R}_{2}-\mathbf{R}_{1}\right| \leqslant 2 a$. We are therefore led to suspect that in higher orders the dominant contribution will come, as in second order, from the terms corresponding to the full partitions in the bottom row of the lattice and then from the region of space in which the inclusions overlap sequentially in the order in which they appear in the partition. There are $(s-1)$ ! full partitions in our lattice of partitions of $s$ labels and, since their contributions differ only by a relabeling of the variables of integration in 
(3.4), they are all the same. We can therefore write our approximation

$$
\begin{aligned}
\epsilon^{*} \mathbf{1} \approx \epsilon_{\mathrm{ov}}^{*} \mathbf{1} \equiv & \epsilon_{1} \mathbf{1}+4 \pi \sum_{s=1}^{\infty} \int_{V_{0}(1|\ldots| s)} \cdots \int d \mathbf{R}_{2} \ldots d \mathbf{R}_{s} \\
& \times b(1|2| \ldots \mid s)(0|\mathrm{C}(1|2| \ldots \mid s)| 0)
\end{aligned}
$$

where the integration is restricted to the overlap region $V_{0}(1|\ldots| s)$ corresponding to the restriction

$$
\left|\mathbf{R}_{j}-\mathbf{R}_{j-1}\right|<2 a, \quad j=2,3, \ldots, s
$$

With this restriction the block distribution function has the simple form

$$
b(1|2| \ldots \mid s)=(-)^{s-1} n^{s} \quad \text { (overlap) }
$$

To see this note that in the sum (2.6) for $b(1|2| \ldots \mid s)$ to each partition obtained from $(1|2| \ldots \mid s)$ by removing slashes there corresponds a product of partial distribution functions at least one of which vanishes on account of the nonoverlap condition. Thus, in the example (2.7) if 1 and 2 overlap and 2 and 3 overlap, $n(1,2,3)=n(1,2)=n(2,3)=0$, and $b(1|2| 3)$ $=n(1) n(2) n(3) \equiv n^{3}$. The chain operator appearing in (3.5) is, using (2.12),

$$
\mathrm{C}(1|2| \ldots \mid s)=\mathrm{M}(1) \cdot \mathrm{L}(2) \cdot \ldots \cdot \mathrm{L}(s)
$$

We thus find

$$
\begin{aligned}
\epsilon_{\mathrm{ov}}^{*} \mathbf{1} \approx & \epsilon_{1} \mathbf{1}+4 \pi \sum_{s=1}^{\infty}(-)^{s-1} n^{s} \int_{V_{0}(1|2| \ldots \mid s)} \cdots \int d \mathbf{R}_{2} \ldots d \mathbf{R}_{s} \\
& \times(O|\mathrm{M}(1) \cdot L(2) \cdot \ldots \cdot \mathrm{L}(s)| 0)
\end{aligned}
$$

where the integration is restricted by the overlap condition (3.6).

The integrals in (3.9) can be performed sequentially starting with $\mathbf{R}_{s}$. To see this, imagine a single inclusion placed with its center at $\mathbf{R}_{s}$, in a uniform field $\mathbf{E}_{0}$. The resulting induced electric field, within and without the inclusion, is

$$
\mathbf{E}_{\text {ind }}(s)=\mathbf{E}(s)-\mathbf{E}_{0}=\mathrm{L}(s) \cdot \mathbf{E}_{0}
$$

as we see from (2.8) and the cluster expansion (2.9). Now in II, Eq. (3.27), we showed that when this induced field is evaluated at a point $\mathbf{r}$ within a sphere of radius $a$ centered at $\mathbf{R}_{s-1}$, and then integrated over all positions $\mathbf{R}_{s}$ such that the inclusion overlaps the sphere, one gets the result

$$
\int_{\left|\mathbf{R}_{s}-\mathbf{R}_{s-1}\right|<2 a} d \mathbf{R}_{s} \mathbf{E}_{\text {ind }}(s)=-\frac{4 \pi}{3 \epsilon_{1}} \mathbf{p}(s), \quad 0<\left|\mathbf{r}-\mathbf{R}_{s-1}\right|<a
$$

where $\mathbf{p}(s)$ is the dipole moment induced in a single inclusion placed in a uniform applied field $\mathbf{E}_{0}$. This is independent of $\mathbf{R}_{s}$ and given by the 
familiar formula

$$
\mathbf{p}(s)=\alpha \mathbf{E}_{0}
$$

where $\alpha$ is the dipole polarizability of the inclusion. Thus, within the inclusion centered at $\mathbf{R}_{s-1}$, the induced field corresponding to an inclusion centered at $\mathbf{R}_{s}$ and integrated over all overlapping configurations is just

$$
\int_{\left|\mathbf{R}_{s}-\mathbf{R}_{s-1}\right|<2 a} d \mathbf{R}_{s} \mathbf{E}_{\text {ind }}(s)=-\frac{4 \pi \alpha}{3 \epsilon_{1}} \mathbf{E}_{0}
$$

This is again a uniform field, so that we may repeat the argument $s-1$ times to obtain

$$
\begin{aligned}
& \int_{V_{0}(1|2| \ldots \mid s)} \cdots \int d \mathbf{R}_{2} \ldots d \mathbf{R}_{s} \mathrm{M}(1) \cdot \mathrm{L}(2) \cdot \ldots \mathrm{L}(s) \cdot \mathbf{E}_{0} \\
& =\left(-\frac{4 \pi \alpha}{3 \epsilon_{1}}\right)^{s-1} \mathrm{M}(1) \cdot \mathbf{E}_{0}
\end{aligned}
$$

But $M(1) \cdot \mathbf{E}_{0}$ is the polarization induced within a single inclusion centered at $\mathbf{R}_{1}$ in a uniform field $\mathbf{E}_{0}$. When this is integrated over $\mathbf{r}$ we get the dipole moment $\alpha \mathbf{E}_{0}$ induced in the inclusion. This integration is just what is done to form the matrix element in (3.9) so that, using that $\mathbf{E}_{0}$ is arbitrary, we get the result

$$
\epsilon_{\mathrm{ov}}^{*}=\epsilon_{1}+4 \pi n \alpha \sum_{s=1}^{\infty}\left(\frac{4 \pi n \alpha}{3 \epsilon_{1}}\right)^{s-1}=\epsilon_{1} \frac{3 \epsilon_{1}+8 \pi n \alpha}{3 \epsilon_{1}-4 \pi n \alpha}=\epsilon_{\mathrm{CM}}
$$

where the last equality follows from (3.2). Thus we have found that the overlap contribution gives rise to the Clausius-Mossotti expression for the effective dielectric constant.

We conclude this section with a number of remarks on the motivation and justification of the overlap approximation which we have just shown corresponds exactly to the Clausius-Mossotti formula. In the above discussion we were led to this approximation by noting that in second order the overlap term is the largest contribution and assuming that this would also be the case in higher orders. In the second order the overlap term is a correction to the first-order term in the following respect. Because of the nonoverlap condition the presence of the first inclusion restricts the possible location of a second inclusion. In the first-order term this "error" was made in the overlap region and therefore the correction is restricted to that region. As we have seen above, it takes the form of the induced field due to the second sphere in the overlap region. In higher orders the terms selected in the overlap approximation represent similar corrections. In each order they correct an "error" made in neglecting the nonoverlap condition in the previous order. 
In second order the term neglected in the overlap approximation, the term denoted by $\epsilon^{*}(1,2)$, corresponds to the change in the polarizability of an inclusion due to the presence of a second inclusion in nonoverlapping configurations. This change is due to the electrical interaction between the inclusions. If in higher orders we were to neglect all such electrical interactions this would correspond in (3.3) to keeping only those terms corresponding to full partitions, from the bottom row of the lattice. That is, the approximation in which electrical interactions are neglected corresponds to (3.5) but without the overlap restriction on the integration. In second order this makes no difference since, as we showed in II, the nonoverlap contribution to $\epsilon^{*}(1 \mid 2)$ vanishes identically. But in higher orders this is not the case. The overlap restriction there implies the neglect of all statistical correlations, i.e., assuming that the partial distribution functions have the form

$$
n(1,2, \ldots, s)= \begin{cases}0, & \text { if }\left|\mathbf{R}_{j}-\mathbf{R}_{k}\right|<2 a \text { for any } j \neq k=1,2, \ldots, s \\ n^{2}, & \text { otherwise }\end{cases}
$$

Then it is a simple matter to show that $b(1|2| \ldots \mid s)$ vanishes except for the configurations corresponding to the overlap condition (3.6). We see therefore that the overlap approximation (3.5), which we have shown corresponds to the Clausius-Mossotti formula, is equivalent to (a) neglecting electrical interactions between the inclusions as well as (b) neglecting in third- and higher-order statistical correlations in the distribution of inclusions. When put this way the Clausius-Mossotti formula seems to be a very severe approximation whose accuracy is surprising.

In this connection it is of interest to remark upon the similarity of our selection of terms leading to the Clausius-Mossotti formula and one made in the kinetic theory of gases to obtain higher-density corrections to the Boltzmann equation. In fact, the Enskog theory for a moderately dense gas of hard spheres can be obtained from a formal density expansion of the Liouville equation by selecting in each order of the density just those terms that correct for the error made by neglecting the nonoverlapping condition in previous orders. ${ }^{(12)}$ As a consequence the Enskog theory neglects all dynamical (i.e., momentum) correlations between successive binary collisions, which is in a way analogous to our neglect of electrical interactions between inclusions. Thus in both our electrical case and the Enskog theory one reasons that the nonoverlap condition is more important than subtle correlations arising from the propagation of the interaction, i.e., from the equations of electrostatics in our electrical case or from Newton's equations of motion in the Enskog theory. For the reader who wishes to pursue this further, we should point out that our electrical problem is more closely analogous to the density expansion of the Lorentz-Boltzmann equation 
with the electric field corresponding to the scattering particle and the inclusions corresponding to the fixed scattering centers.

\section{THE WAVE-VECTOR-DEPENDENT SUSCEPTIBILITY}

The effective dielectric constant $\epsilon^{*}$ is a useful concept for average fields whose spatial variation is small. In situations with rapidly varying fields one must take into account the wave-vector dependence of the effective susceptibility tensor $\boldsymbol{\chi}^{*}(\mathbf{q})$, corresponding to the nonlocal character of the relation between $\langle\mathbf{P}\rangle$ and $\langle\mathbf{E}\rangle$. In this section we first approximate $\chi^{*}(\mathbf{q})$ by making the same selection of terms in the cluster expansion (2.16) which led to the Clausius-Mossotti formula in the spatially uniform case. That is, as in Section 3, we select in each order from the sum in (2.16) the terms corresponding to the full partitions in the bottom row of the lattice, and then restrict the integration to the region in which the inclusions overlap sequentially. Thus, as in (3.9), we approximate

$$
\begin{aligned}
\chi^{*}(\mathbf{q}) \approx \chi_{o v}^{*}(\mathbf{q}) \equiv & \sum_{s=1}^{\infty}(-)^{s-1} n^{s} \int_{V_{0}(1|2| \ldots \mid s)} \cdots \int d \mathbf{R}_{2} \ldots d \mathbf{R}_{s} \\
& \times(\mathbf{q}|\mathrm{M}(1) \cdot \mathrm{L}(2) \cdot \ldots \cdot \mathrm{L}(s)| \mathbf{q})
\end{aligned}
$$

where the overlap region $V_{0}(1|2| \ldots \mid s)$ corresponds to the restriction (3.6). We next evaluate this expression for the special case of polarizable point inclusions, i.e., inclusions of the form of a sphere of radius $a$ and dielectric constant $\epsilon_{1}$ with pointlike dipole polarizability $\alpha$ at its center.

When a polarizable point inclusion is placed with its center at $\mathbf{R}_{s}$ in an external field $\mathbf{E}_{0}(\mathbf{r})$, the induced electric field, within and without the inclusion, is that of a point dipole of moment $\mathbf{p}(s)=\alpha \mathbf{E}_{0}\left(\mathbf{R}_{s}\right)$ at the center of the inclusion. If, therefore, we consider a plane wave applied field of the form

$$
\mathbf{E}_{0}(\mathbf{r})=\mathbf{E}_{\mathbf{q}} e^{i \mathbf{q} \cdot \mathbf{r}}
$$

then the induced field due to an inclusion placed at $\mathbf{R}_{s}$ is

$$
\begin{aligned}
\mathbf{E}_{\text {ind }}(s) & \equiv \mathrm{L}(s) \cdot \mathbf{E}_{0} \\
& =\frac{\alpha}{\epsilon_{1}} e^{i \mathbf{q} \cdot \mathbf{R}_{s}}\left[\frac{3 \mathbf{E}_{\mathbf{q}} \cdot\left(\mathbf{r}-\mathbf{R}_{s}\right)\left(\mathbf{r}-\mathbf{R}_{s}\right)-\left(\mathbf{r}-\mathbf{R}_{s}\right)^{2} \mathbf{E}_{\mathbf{q}}}{\left|\mathbf{r}-\mathbf{R}_{s}\right|^{5}}-\frac{4 \pi}{3} \mathbf{E}_{\mathbf{q}} \boldsymbol{\delta}\left(\mathbf{r}-\mathbf{R}_{s}\right)\right] \\
& =\frac{\alpha}{\epsilon_{1}} e^{i \mathbf{q} \cdot \mathbf{R}_{s}} \frac{\partial}{\partial \mathbf{R}_{s}} \frac{\left(\mathbf{r}-\mathbf{R}_{s}\right) \cdot \mathbf{E}_{\mathbf{q}}}{\left|\mathbf{r}-\mathbf{R}_{s}\right|^{3}}
\end{aligned}
$$

In the terms of (4.1) we need the result of integrating this field over positions $\mathbf{R}_{s}$ within a sphere of radius $2 a$ centered at $\mathbf{R}_{s-1}$ and, since the 
polarizability of an inclusion centered at $\mathbf{R}_{s-1}$ is pointlike, evaluated at $\mathbf{r}=\mathbf{R}_{s-1}$. We therefore calculate

$$
\begin{aligned}
\int_{\left|\mathbf{R}_{s}-\mathbf{R}_{s-1}\right|<2 a} d \mathbf{R}_{s}\left\{\mathbf{E}_{\mathrm{ind}}(s)\right\}_{\mathbf{r}=\mathbf{R}_{s-1}} \\
=-\frac{\alpha}{\epsilon_{1}} e^{i \mathbf{q} \cdot \mathbf{R}_{s-1}} \int_{R<2 a} d \mathbf{R} e^{i \mathbf{q} \cdot \mathbf{R}} \frac{\partial}{\partial \mathbf{R}} \frac{\mathbf{R} \cdot \mathbf{E}_{\mathbf{q}}}{R^{3}} \\
\quad=-\frac{4 \pi \alpha}{\epsilon_{1}} e^{i \mathbf{q} \cdot \mathbf{R}_{s-1}}\left\{\left[1-2 \frac{j_{1}(z)}{z}\right] \hat{q} \cdot \mathbf{E}_{\mathbf{q}} \hat{q}+\frac{j_{1}(z)}{z}\left(\mathbf{E}_{\mathbf{q}}-\hat{q} \cdot \mathbf{E}_{\mathbf{q}} \hat{q}\right)\right\}
\end{aligned}
$$

where $j_{1}(z)=\sin z / z^{2}-\cos z / z$ is the spherical Bessel function, $z=2 q a$, and $\hat{q}=\mathbf{q} / q$. Repeating this $s-1$ times we obtain

$$
\begin{gathered}
\int_{V_{0}(1|2| \ldots \mid s)} \cdots \int d \mathbf{R}_{2} \ldots d \mathbf{R}_{s}\left\{\mathbf{L}(2) \cdot \ldots \cdot \mathrm{L}(s) \cdot \mathbf{E}_{0}\right\}_{\mathbf{r}=\mathbf{R}_{\mathbf{1}}} \\
=\left(-\frac{4 \pi \alpha}{\epsilon_{1}}\right)^{s-1}\left\{\left[1-2 \frac{j_{1}(z)}{z}\right]^{s-1} \hat{q} \cdot \mathbf{E}_{\mathbf{q}} \hat{q}\right. \\
\left.+\left[\frac{j_{1}(z)}{z}\right]^{s-1}\left(\mathbf{E}_{\mathbf{q}}-\hat{q} \cdot \mathbf{E}_{\mathbf{q}} \hat{q}\right)\right\}
\end{gathered}
$$

Now from the definition (2.11) of the operator $M(1)$ we see that $M(1) \cdot \mathbf{E}$ is the polarization within a single inclusion placed with its center at $\mathbf{R}_{1}$ in a field $\mathbf{E}(\mathbf{r})$. For a polarizable point inclusion this polarization is concentrated at the center so that

$$
\int d \mathbf{r} e^{-i \mathbf{q} \cdot \mathbf{r}} \mathbf{M}(1) \cdot \mathbf{E}=\alpha e^{-i \mathbf{q} \cdot \mathbf{R}_{1}} \mathbf{E}\left(\mathbf{R}_{1}\right)
$$

As we see from the prescription (2.17), this integration is exactly what is needed to form the matrix elements of the terms in (4.1). Therefore, using (4.5) for $\mathbf{E}\left(\mathbf{R}_{1}\right)$ in (4.6),

$$
\begin{aligned}
\chi_{\mathrm{ov}}^{*}(\mathbf{q}) \cdot \mathbf{E}_{\mathbf{q}}=n \alpha \sum_{s=1}^{\infty}\left(\frac{4 \pi n \alpha}{\epsilon_{\mathrm{l}}}\right)^{s-1}\{ & {\left[1-2 \frac{j_{1}(z)}{z}\right]^{s-1} \hat{q} \cdot \mathbf{E}_{\mathbf{q}} \hat{q} } \\
& \left.+\left[\frac{j_{1}(z)}{z}\right]^{s-1}\left(\mathbf{E}_{\mathbf{q}}-\hat{q} \cdot \mathbf{E}_{\mathrm{q}} \hat{q}\right)\right\}
\end{aligned}
$$

The sums are now trivial and, since $\mathbf{E}_{\mathbf{q}}$ is arbitrary, we see we can write in a dyadic notation

$$
\chi_{\mathrm{ov}}^{*}(\mathbf{q})=\chi_{l}^{*}(q) \hat{q} \hat{q}+\chi_{l}^{*}(q)(\mathbf{1}-\hat{q} \hat{q})
$$


where

$$
\chi_{l}^{*}(q)=\frac{n \alpha}{1-\left(4 \pi n \alpha / \epsilon_{1}\right)\left[1-2 j_{1}(z) / z\right]}
$$

is the longitudinal susceptibility and

$$
\chi_{t}^{*}(q)=\frac{n \alpha}{1-\left(4 \pi n \alpha / \epsilon_{1}\right)\left[j_{1}(z) / z\right]}
$$

is the transverse susceptibility, with $z=2$ qa. The expression (4.8) is our desired explicit form for the overlap contribution to the susceptibility tensor for the case of polarizable point inclusions.

In the limit of small $q$, using $\lim _{z \rightarrow 0} j_{1}(z) / z=1 / 3$, we see that

$$
\chi_{l}^{*}(0)=\chi_{t}^{*}(0)=\frac{n \alpha}{1-\left(4 \pi n \alpha / 3 \epsilon_{1}\right)}
$$

Putting this in (3.1) we recover the Clausius-Mossotti result (3.15).

\section{POTENTIAL OF A POINT CHARGE}

The average (over the distribution of inclusions) electrostatic potential due to a point charge is the fundamental electrostatic problem for the effective medium, in the sense that the potential due to an arbitrary distribution of charge can be expressed as an integral of the potential of a point charge. In this section we use the approximate formula for the wave-vector-dependent susceptibility to calculate this fundamental solution. For a point charge source the electrostatic equation in the effective medium is

$$
\operatorname{div}\langle\mathbf{D}\rangle=4 \pi Q \delta(\mathbf{r})
$$

where $Q$ is the charge (placed at the origin) and

$$
\langle\mathbf{D}\rangle=\epsilon_{1}\langle\mathbf{E}\rangle+4 \pi\langle\mathbf{P}\rangle
$$

is the dielectric displacement. The electrostatic potential $\langle\phi\rangle$ is introduced in the usual way:

$$
\langle\mathbf{E}\rangle=-\operatorname{grad}\langle\phi\rangle
$$

Here, as introduced in Section $2,\langle>$ means average over the distribution of inclusions. We express $\langle\phi\rangle$ as a Fourier integral

$$
\langle\phi(\mathbf{r})\rangle=\int d \mathbf{q} e^{i \mathbf{q} \cdot \mathbf{r}}\left\langle\phi_{\mathbf{q}}\right\rangle
$$

Then using the above relations together with (2.15) we find after a few steps

$$
\left\langle\phi_{\mathbf{q}}\right\rangle=\frac{Q}{2 \pi^{2}} \frac{1}{q^{2}\left[\epsilon_{1}+4 \pi \chi_{l}^{*}(q)\right]}
$$


where $\chi_{I}^{*}(q)$ is the longitudinal susceptibility. Putting this result in (5.4) and integrating over the directions of $\mathbf{q}$, we find

$$
\langle\boldsymbol{\phi}(\mathbf{r})\rangle=\frac{Q}{r} \cdot \frac{2}{\pi} \int_{0}^{\infty} d q \frac{\sin q r}{q\left[\epsilon_{1}+4 \pi \chi_{l}^{*}(q)\right]}
$$

In the integrand of this expression we can write

$$
\frac{1}{\epsilon_{1}+4 \pi \chi_{l}^{*}(q)}=\frac{1}{\epsilon_{1}+4 \pi \chi_{l}^{*}(0)}+\left[\frac{1}{\epsilon_{1}+4 \pi \chi_{l}^{*}(q)}-\frac{1}{\epsilon_{1}+4 \pi \chi_{l}^{*}(0)}\right]
$$

For the first term we can use the result

$$
\int_{0}^{\infty} d q \frac{\sin q r}{q}=\frac{\pi}{2}
$$

while for the second term we write

$$
\sin q r=\frac{1}{2 i}\left(e^{i q r}-e^{-i q r}\right)
$$

and make the transformation $q \rightarrow-q$ in the integration of the second term in this last expression. Since $\chi_{l}^{*}(q)$ is an even function of $q$, we obtain the expression

$$
\langle\phi(\mathbf{r})\rangle=\frac{Q}{\epsilon_{\mathrm{CM}} r}+\frac{Q}{i \pi r} \int_{-\infty}^{\infty} d q \frac{e^{i q r}}{q}\left[\frac{1}{\epsilon_{1}+4 \pi \chi_{l}^{*}(q)}-\frac{1}{\epsilon_{1}+4 \pi \chi_{l}^{*}(0)}\right]
$$

where we have used (4.11) to express the first term in terms of the Clausius-Mossotti dielectric constant.

In (5.10) the first term is the potential in a medium with dielectric constant $\epsilon_{\mathrm{CM}}$, the second represents therefore corrections due to the nonlocal response described by the wave-vector-dependent susceptibility. To evaluate this term we use the result (4.9) and the expression (3.2) for $\epsilon_{\mathrm{CM}}$ to write

$$
\langle\phi\rangle=\frac{Q}{\epsilon_{\mathrm{CM}} r}+\frac{2 Q\left(\epsilon_{\mathrm{CM}}-\epsilon_{1}\right)^{2}}{i \pi \epsilon_{1} \epsilon_{\mathrm{CM}} r} \int_{-\infty}^{\infty} d z \frac{\left[3 j_{1}(z) / z-1\right] \exp (i z r / 2 a)}{z\left[\epsilon_{\mathrm{CM}}+2 \epsilon_{1}+6\left(\epsilon_{\mathrm{CM}}-\epsilon_{1}\right) j_{1}(z) / z\right]}
$$

where we have made the substitution $z=2 q a$. The integral can be evaluated by the method of residues. ${ }^{(13)}$ Since the integrand vanishes far in the upper half $z$ plane, the path of integration can be extended to a closed path enclosing the upper half plane. The integrand is analytic in the upper half 
plane except for poles at the zeros of the denominator (but not at $z=0$ since the numerator vanishes there). The integral is therefore equal to $2 \pi i$ times the sum of the residues at the points $z_{j}$ in the upper half plane for which

$$
\epsilon_{\mathrm{CM}}+2 \epsilon_{1}+6\left(\epsilon_{\mathrm{CM}}-\epsilon_{1}\right) j_{1}(z) / z=0
$$

Using this equation and the formula $(d / d z)\left[j_{1}(z) / z\right]=-j_{2}(z) / z$, we find we can write the result in the simple form

$$
\langle\phi\rangle=\frac{Q}{\epsilon_{\mathrm{CM}} r}+\frac{Q}{\epsilon_{1} r} \sum_{j} \frac{\exp \left(i z_{j} r / 2 a\right)}{j_{2}\left(z_{j}\right)}
$$

where the sum is over the roots of (5.12) with $\operatorname{Im} z_{j}>0$. Note that if $z_{j}$ is such a root, so is $-\bar{z}_{j}$ (the bar denotes complex conjugate) so that the sum in (5.13) is in fact real, as it must be.

The zeros of (5.12) can be ordered according to their real part. In Table I we give the first five zeros, $z_{j}=x_{j}+i y_{j}$, for various values of the quantity $\left(\epsilon_{\mathrm{CM}}-\epsilon_{1}\right) /\left(\epsilon_{\mathrm{CM}}+2 \epsilon_{1}\right)$. In addition to the zeros shown there, there are (as remarked above) zeros with the real part changed in sign. For fixed $\epsilon_{\mathrm{CM}} / \epsilon_{1}$, the zeros shown in Table I move with increasing $j$ rapidly to the right and up in the $z$ plane. In fact, it is not difficult using (5.12) to show that for large $j$,

$$
z_{j} \sim \begin{cases}2 j \pi+i \ln \frac{(2 j \pi)^{2}\left(\epsilon_{\mathrm{CM}}+2 \epsilon_{1}\right)}{3\left(\epsilon_{\mathrm{CM}}-\epsilon_{1}\right)}, & \epsilon_{1}<\epsilon_{\mathrm{CM}}<\infty \\ (2 j+1) \pi+i \ln \frac{(2 j+1)^{2} \pi^{2}\left(\epsilon_{\mathrm{CM}}+2 \epsilon_{1}\right)}{3\left(\epsilon_{1}-\epsilon_{\mathrm{CM}}\right)}, & 0<\epsilon_{\mathrm{CM}}<\epsilon_{\mathrm{I}}\end{cases}
$$

With this result we can demonstrate convergence of the series (5.13), although we shall not do so here.

The sum in (5.13) vanishes rapidly for $r \gg 2 a$, so the potential is for large $r$ the same as that in a medium with dielectric constant $\epsilon_{\mathrm{CM}}$. The way in which this happens is shown in Fig. 1, where the ratio of $\langle\phi\rangle$ to its asymptotic form is plotted vs. $r$. We have chosen there the rather extreme value $\epsilon_{\mathrm{CM}}=5.5 \epsilon_{1}$, corresponding to $\left(\epsilon_{\mathrm{CM}}-\epsilon_{1}\right) /\left(\epsilon_{\mathrm{CM}}+2 \epsilon_{1}\right)=0.6$, for purposes of illustration to show the oscillations more clearly. For smaller $\epsilon_{\mathrm{CM}}$ the result is qualitatively the same: the mean potential $\langle\boldsymbol{\phi}\rangle$ oscillates about its asymptotic form with the oscillations rapidly damped for $r \gg 2 a$, the amplitude of the oscillations is, however, smaller for smaller $\epsilon_{C M}$. Although we have derived these results using the overlap approximation for $\boldsymbol{\chi}^{*}(\mathbf{q})$, we believe that for $r / 2 a$ not too small they are a qualitatively, and perhaps even quantitatively, accurate representation of the effects of nonlocality. 


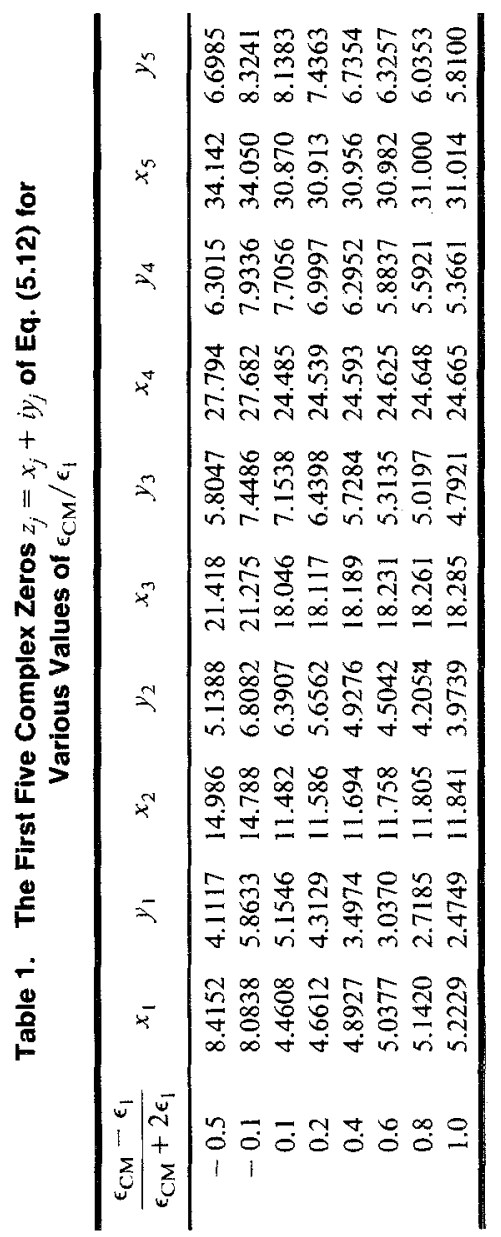




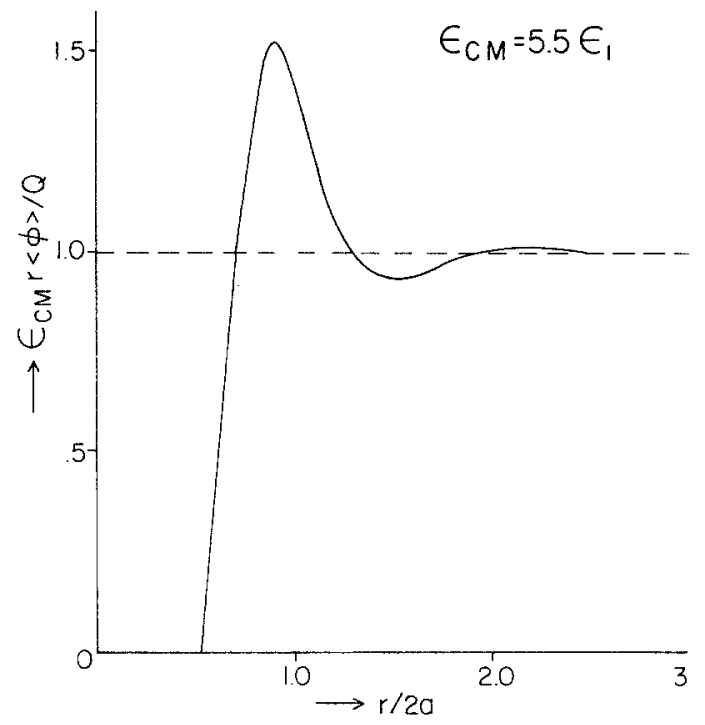

Fig. 1. The potential of a point charge, plotted as the ratio to its asymptotic form: $\langle\phi\rangle \sim Q / \epsilon_{\mathrm{CM}} r$. The medium is a random suspension of hard spheres of radius $a$ and having only dipole polarizability $\alpha$. The potential is calculated in the overlap approximation, as described in the text, and for a value of the density of inclusions such that Eq. (3.2) yields $\epsilon_{\mathrm{CM}}=5.5$.

For $r \ll 2 a$ we are less convinced of the accuracy of the overlap approximation. That is one reason we have not shown that portion of the curve in Fig. 1. Another is that the convergence of the series (5.13) is not so rapid for small $r$. For the portion of the curve shown in Fig. 1, and down to $r / 2 a \approx 0.2$ the first five zeros, given in Table I, give adequate convergence.

Using (5.6) we can show that

$$
\lim _{r \rightarrow 0} r\langle\dot{\phi}\rangle / Q=1 / \epsilon_{l}^{*}(\infty)
$$

where

$$
\epsilon_{l}^{*}(q)=\epsilon_{1}+4 \pi x_{i}^{*}(q)
$$

Using the overlap approximation, where $x_{l}^{*}(q)$ is given by (4.9), we find

$$
\frac{2}{\epsilon_{l}^{*}(\infty)}=\frac{1}{\epsilon_{1}}\left(1-\frac{4 \pi n \alpha}{\epsilon_{1}}\right)=\frac{5 \epsilon_{1}-2 \epsilon_{\mathrm{CM}}}{\epsilon_{1}\left(\epsilon_{\mathrm{CM}}+2 \epsilon_{1}\right)}
$$

It is of interest to compare this result with the exact result for a two-phase 
medium:

$$
\frac{1}{\epsilon_{l}^{*}(\infty)}=\frac{\psi}{\epsilon_{2}}+\frac{1-\psi}{\epsilon_{1}} \quad \text { (two phase) }
$$

where $\psi$ is the volume fraction occupied by the phase with dielectric constant $\epsilon_{2}$. The result (5.18) follows from the remark that a point charge placed at random in the two-phase medium will have chance $\psi$ of being in a region of dielectric constant $\epsilon_{2}$ and chance $1-\psi$ of being in a region of dielectric constant $\epsilon_{1}$. Applying a corresponding argument to our polarizable point model of the inclusion, that of hard spheres of dielectric constant $\epsilon_{1}$ with pointlike polarizability $\alpha$ at the center, one might expect $1 / \epsilon_{l}^{*}(\infty)$ $=1 / \epsilon_{1}$, since a point charge placed at random in the medium will almost surely find itself in a region of dielectric constant $\epsilon_{1}$. The deviation of the result (5.17) from this form reflects the singular nature of our model.

We conclude this section with some remarks upon our results in relation with the singular points of the Clausius-Mossotti theory.

The case where the quantity $\left(\epsilon_{\mathrm{CM}}-\epsilon_{1}\right) /\left(\epsilon_{\mathrm{CM}}+2 \epsilon_{1}\right)$ tends to unity, i.e., where $\epsilon_{\mathrm{CM}}$ is infinite, may be regarded as the critical point of the ClausiusMossotti theory. ${ }^{(14)}$ At this point, as we see from the corresponding entries in Table I, there is no singular behavior in the contributions to the potential arising from the nonlocal response, the sum in (5.13). On the other hand, at this point the first term in (5.13) vanishes, the expected result for the potential in a local medium with an infinite dielectric constant. We conclude that this is only a critical point for the local Clausius-Mossotti theory, there is no singular behavior in the nonlocal corrections to the response.

If we extend the series expression (5.13) for $\langle\phi\rangle$ to values of $\left(\epsilon_{\mathrm{CM}}-\epsilon_{1}\right)$ $/\left(\epsilon_{\mathrm{CM}}+2 \epsilon_{1}\right)$ greater than unity, corresponding to unphysical negative values of $\epsilon_{\mathrm{CM}}$, the imaginary part of the zeros $z_{j}$ continues to decrease, until a critical value $\left(\epsilon_{\mathrm{CM}}-\epsilon_{1}\right) /\left(\epsilon_{\mathrm{CM}}+2 \epsilon_{1}\right)=5.8024$ is reached, at which $z_{1}=$ 5.7634 is pure real. The higher zeros corresponding to this critical value have finite imaginary parts so their contribution to the sum vanishes for large $r$, leaving the oscillatory, undamped, contribution of $z_{1}$. Since this value of $z_{1}$ is a zero of $j_{2}(z)$, we see from (5.13) that the amplitude of this contribution diverges at the critical value. An exactly similar situation occurs when we extend the expression (5.13) to large negative values of the above quantity. Then there is another critical value $\left(\epsilon_{\mathrm{CM}}-\epsilon_{1}\right) /\left(\epsilon_{\mathrm{CM}}+2 \epsilon_{1}\right)$ $=-43.19$, at which $z_{1}=9.095$ is again real. We may regard this singular behavior as corresponding to critical points of the nonlocal theory. We therefore have the interesting situation in which there are three critical points, one for the local theory when the quantity $\left(\epsilon_{\mathrm{CM}}-\epsilon_{1}\right) /\left(\epsilon_{\mathrm{CM}}+2 \epsilon_{1}\right)$ is unity, and two for the nonlocal response when this quantity takes the value 
5.8024 and -43.19 , respectively. Of course these results are a consequence of our overlap approximation, but we would expect their qualitative features to be unchanged in an exact theory.

Some years ago Diener and Käseberg ${ }^{(15)}$ extended the coherent potential approximation (CPA) to calculate the effective wave-vector-dependent dielectric constant $\epsilon_{l}^{*}(q)$ of a two-phase medium. The coherent potential approximation is a self-consistent theory whose physical basis is not entirely clear, but which in the local theory (i.e., $q=0$ ) has the advantages that it yields an expression for the effective dielectric constant which is symmetric in the two phases and which has the correct Clausius-Mossotti form at the two extremes of low concentrations of one phase or the other. ${ }^{(16)}$ The Diener and Käseberg extension to finite $q$ has the same advantages. Their result, however, is in the form of an implicit equation for $\epsilon_{l}^{*}(q)$, which complicates the discussion. Nevertheless, although they did not calculate the potential of a point charge, they found in their expression an indication of a strong increase in the range of the nonlocal response when $\epsilon_{1}$ and $\epsilon_{2}$ are very different, just as we have found. Since they did not consider unphysical values of $\epsilon_{1}$ and $\epsilon_{2}$, they did not find nonlocal dielectric critical points such as we have discussed above.

In a recent paper Diener and Weissbarth ${ }^{(17)}$ have derived upper and lower bounds for $\epsilon^{*}(q)$ and have studied these bounds for binary cell mixtures. Similarly, in future work we hope to compare our results with the bounds for a system of spherical inclusions. The second-order bound contains the two-point correlation function and hence requires more statistical input than our extension of the Clausius-Mossotti formula.

\section{ACKNOWLEDGMENT}

We are indebted to the NATO Research Grants Program, Grant No. 1945 , for support for this work.

\section{REFERENCES}

1. V. M. Agranovich and V. L. Ginzburg, Spatial Dispersion in Crystal Optics and the Theory of Excitons (Interscience, New York, 1966).

2. G. D. Mahan and R. M. Mazo, Phys. Rev. 175:1191 (1968).

3. L. M. Hafkenscheid and J. Vlieger, Physica 75:57 (1974).

4. A. Mead, Phys. Rev. B 17:4644 (1978).

5. J. J. Hopfield and D. G. Thomas, Phys. Rev. 132:563 (1963).

6. B. U. Felderhof, G. W. Ford, and E. G. D. Cohen, J. Stat. Phys. 28: 135 (1982), referred to as $\mathrm{I}$.

7. B. U. Felderhof, G. W. Ford, and E. G. D. Cohen, J. Stat. Phys. 28:649 (1982), referred to as II. 
8. C. J. F. Böttcher, Theory of Electric Polarization, Vol. I, 2nd ed. (Elsevier, Amsterdam, 1973), p. 172.

9. G. D. Mahan, Solid State Ionics 1:29 (1980).

10. Z. Hashin and S. Shtrikman, J. Appl. Phys. 33:3125 (1962).

11. B. U. Felderhof, J. Phys. C 15:1731, 3943, 3953 (1982).

12. J. R. Dorfman and E. G. D. Cohen, J. Math. Phys. 81:288 (1967).

13. E. T. Whittaker and G. N. Watson, Modern Analysis, 4th ed. (Cambridge University Press, Cambridge, 1952), Chap. VI.

14. C. J. F. Böttcher, Theory of Electric Polarization, Vol. 1, 2nd ed. (Elsevier, Amsterdam, 1973), p. 200.

15. G. Diener and F. Käseberg, Int. J. Solids Structures 12:173 (1976).

16. D. A. G. Bruggeman, Ann. Phys. (Leipzig) 24:636 (1935).

17. G. Diener and J. J. Weissbarth, Continuum Models of Discrete Systems 4, O. Brulin and R. K. T. Hsieh, eds. (North-Holland, Amsterdam, 1981), p. 349. 